\title{
Length and PKA Dependence of Force Generation and Loaded Shortening in Porcine Cardiac Myocytes
}

\author{
Kerry S. McDonald, ${ }^{1}$ Laurin M. Hanft, ${ }^{1}$ Timothy L. Domeier, ${ }^{1}$ and Craig A. Emter ${ }^{2}$ \\ ${ }^{1}$ Department of Medical Pharmacology \& Physiology, School of Medicine, University of Missouri, Columbia, MO 65212, USA \\ ${ }^{2}$ Department of Biomedical Sciences, College of Veterinary Medicine, University of Missouri, Columbia, MO 65212, USA
}

Correspondence should be addressed to Kerry S. McDonald, mcdonaldks@missouri.edu

Received 20 February 2012; Accepted 1 May 2012

Academic Editor: John Konhilas

Copyright (C) 2012 Kerry S. McDonald et al. This is an open access article distributed under the Creative Commons Attribution License, which permits unrestricted use, distribution, and reproduction in any medium, provided the original work is properly cited.

\begin{abstract}
In healthy hearts, ventricular ejection is determined by three myofibrillar properties; force, force development rate, and rate of loaded shortening (i.e., power). The sarcomere length and PKA dependence of these mechanical properties were measured in porcine cardiac myocytes. Permeabilized myocytes were prepared from left ventricular free walls and myocyte preparations were calcium activated to yield $\sim 50 \%$ maximal force after which isometric force was measured at varied sarcomere lengths. Porcine myocyte preparations exhibited two populations of length-tension relationships, one being shallower than the other. Moreover, myocytes with shallow length-tension relationships displayed steeper relationships following PKA. Sarcomere length- $K_{\text {tr }}$ relationships also were measured and $K_{\text {tr }}$ remained nearly constant over $\sim 2.30 \mu \mathrm{m}$ to $\sim 1.90 \mu \mathrm{m}$ and then increased at lengths below $1.90 \mu \mathrm{m}$. Loaded-shortening and peak-normalized power output was similar at $\sim 2.30 \mu \mathrm{m}$ and $\sim 1.90 \mu \mathrm{m}$ even during activations with the same $\left[\mathrm{Ca}^{2+}\right]$, implicating a myofibrillar mechanism that sustains myocyte power at lower preloads. PKA increased myocyte power and yielded greater shortening-induced cooperative deactivation in myocytes, which likely provides a myofibrillar mechanism to assist ventricular relaxation. Overall, the bimodal distribution of myocyte length-tension relationships and the PKAmediated changes in myocyte length-tension and power are likely important modulators of Frank-Starling relationships in mammalian hearts.
\end{abstract}

\section{Introduction}

The primary role of cardiac myocytes is to develop force and power. In the isovolumic phase of the cardiac cycle, left ventricular myocytes develop (near)-isometric force against the enclosed ventricular chamber and in doing so increases ventricular pressure. When ventricular pressure exceeds aortic pressure the aortic valve opens, and myocyte force production is accompanied by loaded shortening (i.e., power) as blood is ejected from the ventricle into the systemic circulation. The rate of myocyte force generation determines the duration of isovolumic ventricular contraction and, consequently, the amount of the cardiac cycle devoted to ejection. The blood volume ejected per beat is determined by chamber compression, which is governed by (i) systolic ejection time, (ii) the number of force generating cross-bridges (which controls where on the force-velocity relation the ensemble of cross-bridges will work), and (iii) the inherent rate of loaded cross-bridge cycling. Hence, ventricular ejection is highly dependent on three myofibrillar mechanical properties: (1) force, (2) rate of force development, and (3) rate of loaded shortening (i.e., myocyte power output). There is considerable information related to these cardiac myofibrillar contractile properties in rodents [1-11], and these biophysical properties, in part, underlie the unique ventricular function of these species when compared to larger animals and humans [12-14]. However, there are fewer studies that have focused on cardiac myofibrillar mechanics in pig, a species of high translational relevance given its anatomic similarities to humans. Most investigations of porcine myofibrillar preparations have focused on steady-state properties at a single sarcomere length $[15,16]$ or examined stretch activation [17]. In this study we investigated three key myofibrillar mechanical properties (i.e., force, rate of force 
development, and loaded shortening) and their dependence on sarcomere length and PKA in porcine left ventricular cardiac myocytes.

\section{Methods}

2.1. Animal Model. Adult male yucatan miniature swine (14 months old) weighing $30-40 \mathrm{~kg}$ were obtained from the breeder (Sinclair Research Center; Columbia, MO). Animal care and use procedures complied with the Guide for the Care and Use of Laboratory Animals issued by the National Research Council and were approved by the University of Missouri Animal Care and Use Committee.

2.2. In Vivo Cardiovascular Function. Animals $(n=4)$ were initially anesthetized with a telazol $(5 \mathrm{mg} / \mathrm{kg}) / x y l a z i n e$ $(2.25 \mathrm{mg} / \mathrm{kg})$ mix and maintained using inhaled isoflurane $(\approx 1.75 \%)$. Heparin was given with an initial loading dose of $300 \mathrm{U} / \mathrm{kg}$ IV, followed by maintenance of $100 \mathrm{U} / \mathrm{kg}$ each hour. A median sternotomy was performed and the pericardium was opened near the apex for insertion of the pressurevolume $(P-V)$ loop catheter. $P-V$ loops were measured utilizing a calibrated 7F admittance-based ADVantage catheter (SciSense; London, Ontario, Canada) positioned in the LV. A $14 \mathrm{~F}$ balloon occlusion catheter was advanced to the inferior vena cava at the level of the apex of the heart via the deep femoral vein. Peripheral systemic MAP was measured via a fluid filled 6F LCB SH guide catheter (Boston Scientific) introduced through a 7F sheath placed in the right femoral artery and positioned in the descending aorta distal to the aortic band. Catheter placement was visualized and confirmed using angiography (InfiMed software) and Visipaque contrast medium. Following placement of the catheter, animals were brought to a peripheral MAP of $80 \mathrm{mmHg}$ using phenylephrine (I.V. $1-3 \mu \mathrm{g} / \mathrm{kg} / \mathrm{min}$ ) and allowed to stabilize for 5 minutes. $P-V$ loops were collected before and after a single dose of dobutamine $(5 \mu \mathrm{g} / \mathrm{kg} / \mathrm{min}$ I.V.) administered for 5 minutes under conditions of reducing preload achieved through transient occlusion of the inferior vena cava via inflation of the balloon catheter. Our admittance based $P-V$ loop system requires input of baseline stroke volume (SV), which was determined one week prior to the terminal studies using ultrasound and calculated as previously reported [18] using the equation $\mathrm{SV}=\pi(r)^{2} *$ VTI where $r$ is the radius and VTI is the velocity time interval (measured from apical four-chamber view). Aortic radius was calculated from the aortic left ventricular outflow track (measured in parasternal 2D view).

2.3. Isolation of Cardiac Myocytes. The heart was excised from the experimental animal following administration of a preanesthetic mixture of telazol ( $5 \mathrm{mg} / \mathrm{kg}) /$ xylazine $(2.25 \mathrm{mg} /$ $\mathrm{kg}$ ) and permeabilized myocytes were isolated as previously described [19]. Briefly, a section of left ventricular free wall $\left(\sim 10 \mathrm{~cm}^{3}\right)$ near the left anterior descending (LAD) coronary artery was removed and half was rapidly frozen in liquid nitrogen for biochemical analyses, and the other half was placed in ice cold relaxing solution for myocyte experiments.
The piece in relaxing solution was cut into smaller pieces (2$3 \mathrm{~mm}$ ) and homogenized with a Waring blender. The resultant slurry was centrifuged $75 \mathrm{sec}$ at $165 \times \mathrm{g}$ and the pellet was suspended for $3 \mathrm{~min}$ in $0.5 \%$ ultrapure Triton X-100 (Pierce Chemical Co.) in relaxing solution. The permeabilized myocytes were washed and centrifuged twice with cold relaxing solution with the final suspension kept on ice during the day of the experiment.

For intact myocyte isolation, a section of the left-ventricular free wall was perfused via cannulation of the LAD. The tissue was perfused with a nominally calcium-free saline solution containing heparin for 10 minutes, followed by a minimal essential medium (MEM) solution containing $45 \mu \mathrm{g} / \mathrm{mL}$ Liberase Blendzyme TH (Roche Applied Science, Indianapolis, IN, USA) for 30 minutes at $37^{\circ} \mathrm{C}$. Digested tissue was minced and filtered, and the dissociated myocytes were washed and maintained in an MEM solution with $50 \mu \mathrm{M}$ calcium at room temperature until experimental procedures.

2.4. Solutions. Relaxing solution in which the ventricles were disrupted, skinned, and suspended contained (in $\mathrm{mmol} / \mathrm{L}$ ): EGTA 2, $\mathrm{MgCl}_{2}$ 5, ATP 4, imidazole 10, and $\mathrm{KCl} 100$ at $\mathrm{pH}$ 7.0. Compositions of relaxing and activating solutions used in mechanical measurements were as follows $(\mathrm{mmol} / \mathrm{L})$ : EGTA 7, $\mathrm{MgCl}_{2}$ 5, imidazole 20, ATP 4, creatine phosphate 14.5, $\mathrm{pH}$ 7.0, $\mathrm{Ca}^{2+}$ concentrations of $10^{-9} \mathrm{M}$ (relaxing solution) and $10^{-4.5} \mathrm{M}$ (maximal activating solution), and sufficient $\mathrm{KCl}$ to adjust ionic strength to $180 \mathrm{mM}$. The final concentrations of each metal, ligand, and metal-ligand complex were determined with the computer program of Fabiato [20]. Immediately preceding activations, muscle preparations were immersed for $60 \mathrm{~s}$ in a solution of reduced $\mathrm{Ca}^{2+}$ EGTA buffering capacity, identical to normal relaxing solution except that EGTA is reduced to $0.5 \mathrm{mM}$. This protocol resulted in more rapid steady-state force development and helped preserve the striation pattern during activation. Intact cardiomyocyte experiments were performed in a physiological saline solution containing (in $\mathrm{mM}$ ). $135 \mathrm{NaCl}, 4 \mathrm{KCl}, 2$ $\mathrm{CaCl}_{2}, 1 \mathrm{MgCl}_{2}, 10$ D-glucose, 10 Hepes, pH 7.4 with $\mathrm{NaOH}$.

2.5. Experimental Apparatus. The experimental apparatus for physiological measurements of myocyte preparations was similar to one previously described in detail [21] and modified for cardiac myocyte preparations [7]. Myocyte preparations were attached between a force transducer and torque motor by placing the ends of the myocyte preparation into stainless steel troughs ( 25 gauge). The ends of the myocyte preparations were secured by overlaying a $0.5 \mathrm{~mm}$ length of 3-0 monofilament nylon suture (Ethicon, Inc.) onto each end of the myocyte, and then tying the suture into the troughs with two loops of 10-0 monofilament (Ethicon, Inc). The attachment procedure was performed under a stereomicroscope ( $\sim 100 \mathrm{x}$ magnification) using finely shaped forceps.

Prior to mechanical measurements, the experimental apparatus was mounted on the stage of an inverted microscope (model IX-70, Olympus Instrument Co., Japan), which was placed upon a pneumatic vibration isolation table having 


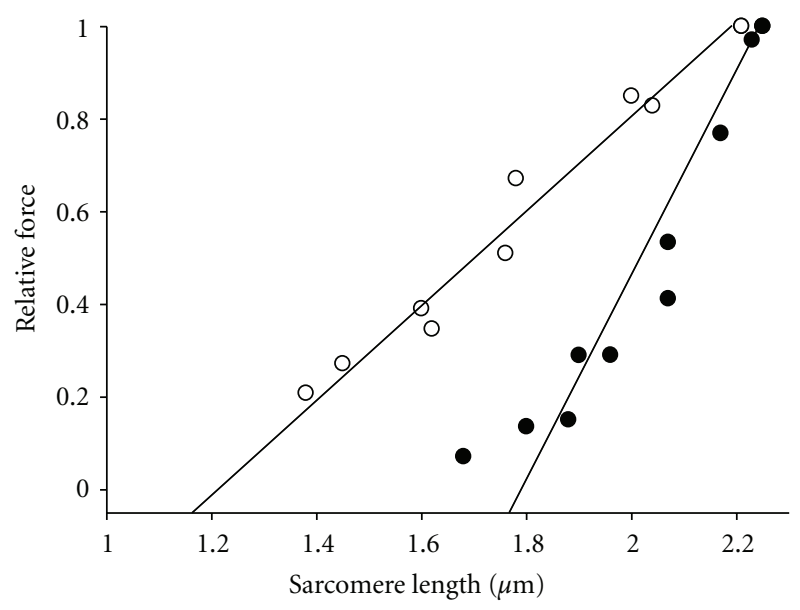

(a)

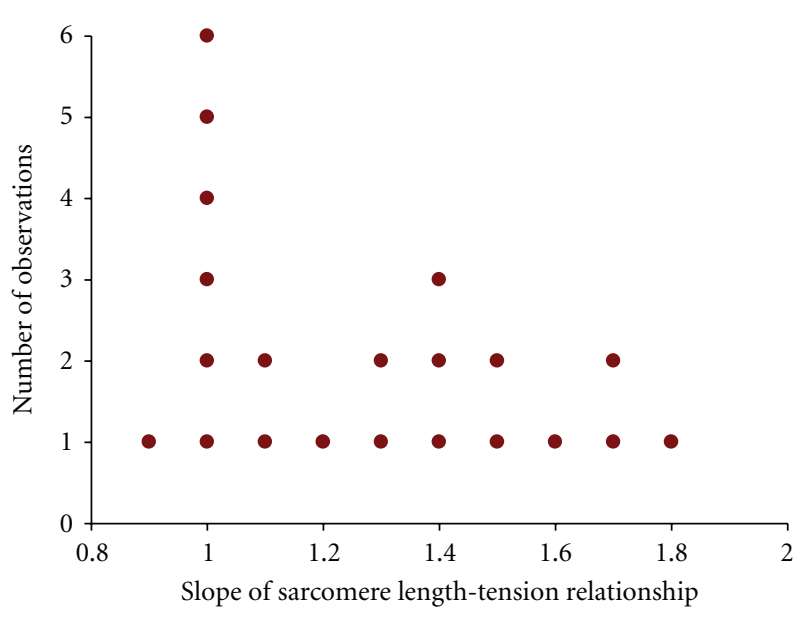

(b)

FIGURE 1: Sarcomere length-tension relationships in porcine skinned ventricular cardiac myocyte preparations. (a) Muscle cell preparations were mounted between a force transducer and motor, calcium activated to yield $~ 50 \%$ maximal force, then isometric force was measured over a range of sarcomere lengths monitored using an IonOptix SarLen system. (b) Histogram showing the slopes of length-tension relationships obtained in porcine cardiac myocytes.

a cut-off frequency of $\sim 1 \mathrm{~Hz}$. Mechanical measurements were performed using a capacitance-gauge transducer (Model 403-sensitivity of $20 \mathrm{mV} / \mathrm{mg}$ (plus a 10x amplifier) and resonant frequency of $600 \mathrm{~Hz}$; Aurora Scientific, Inc., Aurora, ON, Canada). Length changes were introduced using a DC torque motor (model 308, Aurora Scientific, Inc.) driven by voltage commands from a personal computer via a 12-bit D/A converter (AT-MIO-16E-1, National Instruments Corp., Austin, TX, USA). Force and length signals were digitized at $1 \mathrm{kHz}$ and stored on a personal computer using LabView for Windows (National Instruments Corp.). Sarcomere length was monitored simultaneous with force and length measurements using IonOptix SarcLen system (IonOptix, Milton, MA), which used a fast Fourier transform algorithm of the video image of the myocyte. Microscopy was done using a 40x objective (Olympus UWD 40) and a $2.5 \mathrm{x}$ intermediate lens.

2.6. Sarcomere-Length Tension Measurements. All mechanical measurements on cardiac myocytes were performed at $13 \pm 1^{\circ} \mathrm{C}$. For sarcomere length-tension measurements, an experimental protocol was performed similar to previously described [22]. Following attachment of myocyte preparation to the apparatus, the relaxed preparation was adjusted to a sarcomere length of $\sim 2.35 \mu \mathrm{m}$ and then the preparation was maximally $\mathrm{Ca}^{2+}$ activated in pCa 4.5 solution. For sarcomere length-tension measurements, the cell preparation was transferred to a pCa solution that yielded $\sim 50 \%$ maximal (i.e., pCa 4.5 or $\mathrm{P}_{4.5}$ ) force and then isometric force was measured over a range of sarcomere lengths monitored by the IonOptix SarcLen system (IonOptix, Milton, MA). Isometric force and sarcomere length were measured simultaneously. Sarcomere length was adjusted in a range between $\sim 2.35 \mu \mathrm{m}$ and to $\sim 1.4 \mu \mathrm{m}$ by manual manipulation of the length micrometer while the preparation was $\mathrm{Ca}^{2+}$ activated. After each sarcomere length change, $\sim 10-15$ seconds were provided to allow for development of steady-state force. Force at each sarcomere length was obtained via a slack-restretch maneuver (see below for description). For analysis, force at each sarcomere length was normalized to the force obtained at sarcomere length $\sim 2.35 \mu \mathrm{m}$ (during the submaximal $\mathrm{Ca}^{2+}$ activation). Since force during submaximal $\mathrm{Ca}^{2+}$ activations invariably rose slightly during the sustained activation, normalized forces were calculated by interpolating force measurements at sarcomere length $2.35 \mu \mathrm{m}$, which were performed at the beginning and end of the series of force measurements. At the end of each experiment, preparations were activated a second time in pCa 4.5 solutions and only experiments in which maximal tension remained $>80 \%$ of initial were used for analysis. To assess the effects of PKA, length-tension relationships were performed before and after 45 min incubation with PKA (Sigma, $0.125 \mathrm{U} / \mu \mathrm{L}$ ). The pCa solution for length tension curves was adjusted to yield the same forces before and after PKA due to decreased $\mathrm{Ca}^{2+}$ sensitivity of force following PKA.

\subsection{Measurement of the Rate of Force Redevelopment, Loaded} Shortening, and Power. Force redevelopment rates were obtained using a procedure previously described for skinned cardiac myocyte preparations [23-25]. While in $\mathrm{Ca}^{2+}$ activating solution, the myocyte preparation was rapidly shortened by $15-20 \%$ of initial length $\left(L_{0}\right)$ to yield zero force. The myocyte preparation was then allowed to shorten for $\sim 20 \mathrm{~ms}$; after 20 ms the preparation was rapidly restretched to $\sim 105 \%$ of its initial length $\left(L_{0}\right)$ for $2 \mathrm{~ms}$ and then returned to $L_{0}$. Tension redevelopment following a slack-restretch maneuver was fit by a single exponential equation:

$$
F=F_{\max }\left(1-e^{-k_{\mathrm{tr}} t}\right)+F_{\text {res }}
$$


TABLE 1: Porcine cardiac myocyte preparation characteristics.

\begin{tabular}{|c|c|c|c|c|c|c|c|}
\hline & $n$ & Length $(\mu \mathrm{m})$ & Width $(\mu \mathrm{m})$ & $\begin{array}{l}\text { Sarcomere length } \\
(\mu \mathrm{m})\end{array}$ & $\begin{array}{l}\text { Passive force } \\
\qquad(\mu \mathrm{N})\end{array}$ & $\begin{array}{l}\text { Maximum force }\left(\mathrm{P}_{4.5}\right) \\
(\mu \mathrm{N})\end{array}$ & $\begin{array}{c}\text { Maximum force }\left(\mathrm{P}_{4.5}\right) \\
\left(\mathrm{kN} \mathrm{m}^{-2}\right)\end{array}$ \\
\hline Cardiac myocytes & 36 & $129 \pm 28$ & $28 \pm 9$ & $2.29 \pm 0.06$ & $0.70 \pm 0.45$ & $30 \pm 14$ & $75 \pm 32$ \\
\hline
\end{tabular}

Values are means \pm S.D.
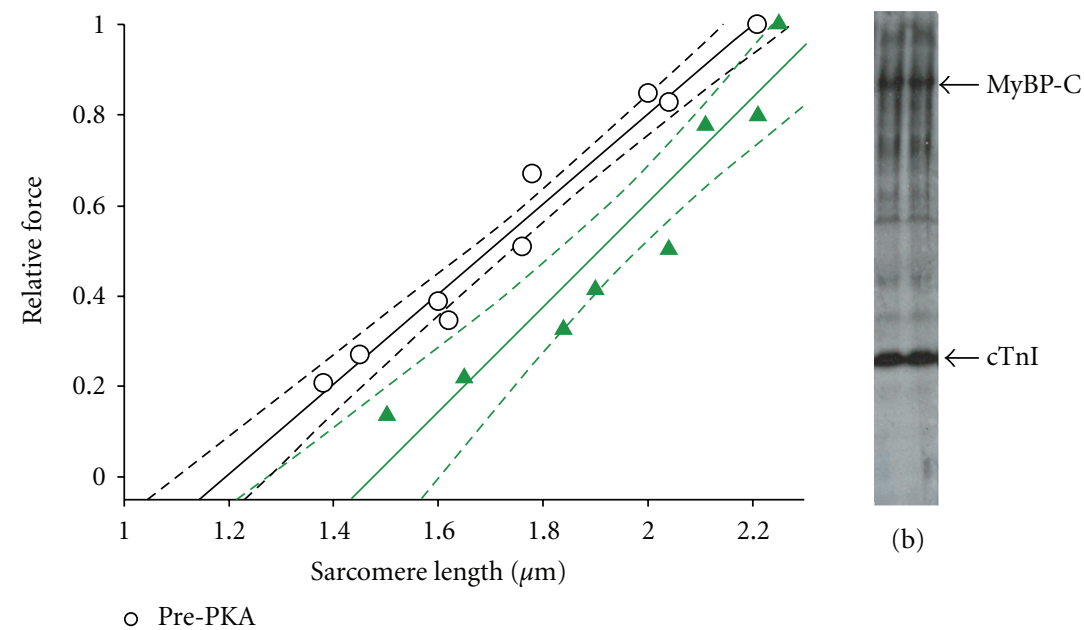

O Pre-PKA

$\triangle$ Post-PKA

(a)

FIGURE 2: (a) Pig cardiac myocyte sarcomere length-tension relationships before and after PKA treatment. PKA-induced phosphorylation markedly steepened the length-tension relationship. (b) An autoradiogram showing radiolabeled phosphate incorporation into pig cardiac myofibrillar proteins (MyBP-C and cTnI) upon PKA treatment. Without PKA treatment, there was no radiolabelled ATP incorporation (data not shown).

where $F$ is force at time $t, F_{\max }$ is maximal force, $k_{\mathrm{tr}}$ is the rate constant of force development, and $F_{\text {res }}$ represents any residual tension immediately after the slack-restretch maneuver.

Power output of single skinned myocyte preparations was determined at varied loads as described earlier [26]. Briefly, myocytes were placed in activating solution and once steady-state force developed, a series of force clamps (less than steady-state force) were performed to determine isotonic shortening velocities. Using a servo-system, force was maintained constant for a designated period of time (200 to $250 \mathrm{msec}$ ) while the length change was continuously monitored. Following the force clamp, the myocyte preparation was slackened to reduce force to near zero to allow estimation of the relative load sustained during isotonic shortening; the myocyte was subsequently re-extended to its initial length.

Myocyte preparation length traces during loaded shortening were fit to a single decaying exponential equation:

$$
L=A e^{-k t}+C
$$

where $L$ is cell length at time $t, A$, and $C$ are constants with dimensions of length, and $k$ is the rate constant of shortening ( $\left.k_{\text {shortening }}\right)$. Velocity of shortening at any given time, $t$, was determined as the slope of the tangent to the fitted curve at that time point. In this study, velocities of shortening were calculated by extrapolation of the fitted curve to the onset of the force clamp (i.e., $t=0$ ).
Hyperbolic force-velocity curves were fit to the relative force-velocity data using the Hill equation [27]:

$$
(P+a)(V+b)=\left(P_{0}+a\right) b
$$

where $P$ is force during shortening at velocity $V, P_{0}$ is the peak isometric force, and $a$ and $b$ are constants with dimensions of force and velocity, respectively. Power-load curves were obtained by multiplying force $x$ velocity at each load on the force-velocity curve. The optimum force for mechanical power output $\left(F_{\text {opt }}\right)$ was calculated using [28]:

$$
F_{\mathrm{opt}}=\left(a^{2}+a * P_{0}\right)^{1 / 2}-a \text {. }
$$

Curve fitting was performed using a customized program written in Qbasic, as well as commercial software (Sigmaplot).

2.8. Intracellular Calcium Measurements. Intact myocytes were plated on laminin coated coverslips and loaded with $5 \mu \mathrm{M}$ of the calcium indicator dye fluo-4/AM for 10 minutes, followed by a 20 -minute wash. 2-dimensional laser-scanning confocal fluorescence microscopy was performed using the resonance scanhead of a Leica SP5 (Leica Microsystems, Buffalo Grove, IL, USA), with excitation at $488 \mathrm{~nm}$ and emission collected from $510-550 \mathrm{~nm}$. Field stimulation $(0.5 \mathrm{~Hz})$ with a pair of platinum electrodes was used to induce action potentials and intracellular calcium transients. To analyze 


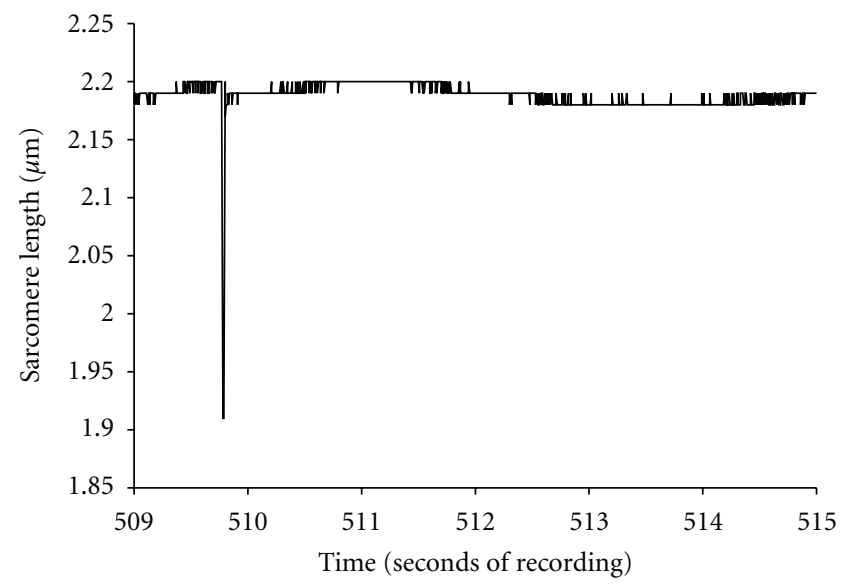

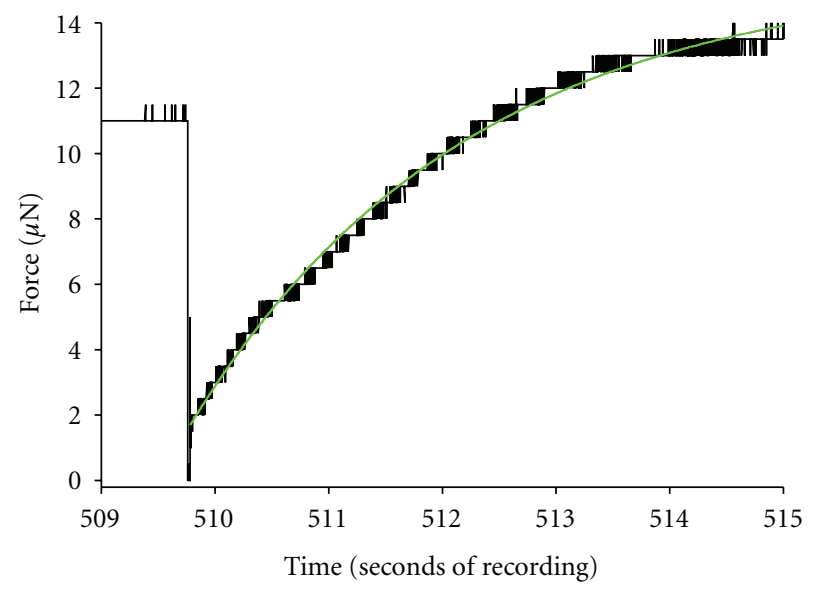

(a)

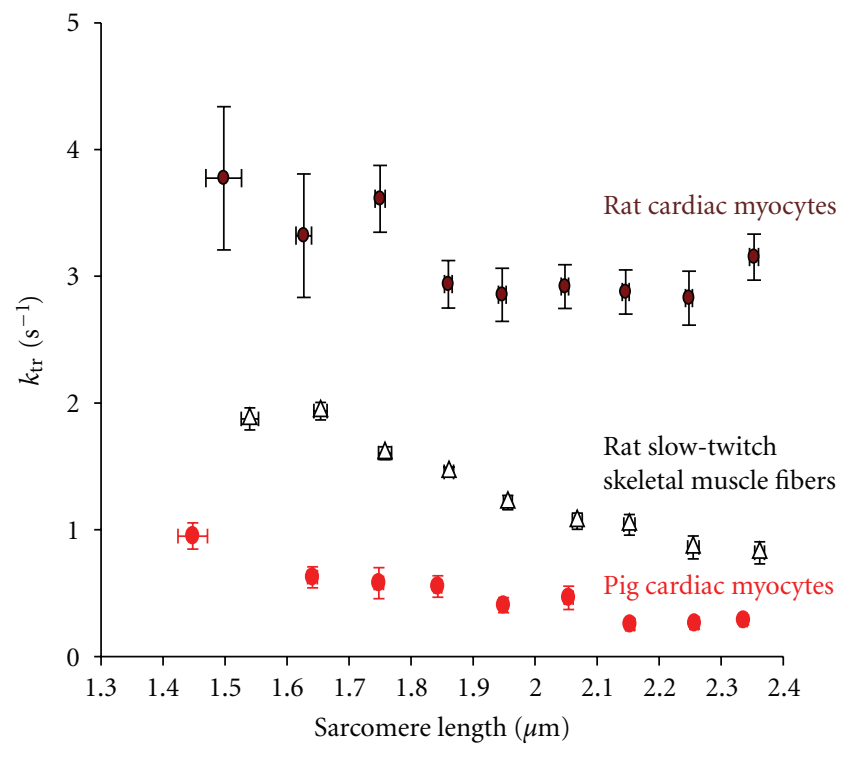

(b)

FIGURE 3: Sarcomere length dependence of the rate constant of force redevelopment $\left(k_{\mathrm{tr}}\right)$. (a) Slow time-based recordings of sarcomere length

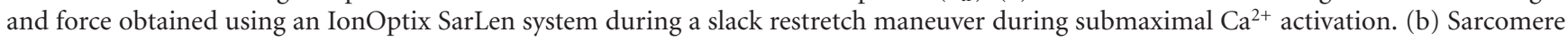
length-dependence of $k_{\text {tr }}$ for rat slow-twitch skeletal muscle fibers, rat cardiac myocytes, and pig cardiac myocytes. Although pig cardiac myocyte $k_{\text {tr }}$ was much slower at all sarcomere lengths compared to rat cardiac myocytes, both pig and rat cardiac cell preparations showed that $k_{\mathrm{tr}}$ increased at short sarcomere lengths despite reductions in force implicating that sarcomere length overrides the Ca ${ }^{2+}$ activation dependence of $k_{\mathrm{tr}}$.

recovery kinetics, calcium transients were normalized using the following formula: $\left[\left(F-F_{\text {baseline }}\right) /\left(F_{\text {peak }}-F_{\text {baseline }}\right)\right]$.

2.9. SDS-PAGE and Autoradiography. The gel electrophoresis procedure was similar to one previously described $[12,29]$. The gels for SDS-PAGE were prepared with a 3.5\% acrylamide stacking gel and a 12\% acrylamide resolving gel. Samples were separated by SDS-PAGE at constant voltage $(250 \mathrm{~V})$ for $8 \mathrm{~h}$. Gels were initially fixed in a 10\% acetic acid-50\% ethanol solution, followed by $2 \%$ glutaraldehyde. MyHC isoforms were visualized by ultrasensitive silver staining, and gels were subsequently dried between mylar sheets.

PKA-induced phosphate incorporation into myofibrillar substrates was determined as described previously [30]. Briefly, skinned cardiac myocytes $(10 \mu \mathrm{g})$ were incubated with the catalytic subunit of PKA $(5 \mu \mathrm{g} / \mathrm{mL})$ and $50 \mu \mathrm{Ci}$ $\left[\gamma-{ }^{32} \mathrm{P}\right]$ ATP at room temperature $\left(21-23^{\circ} \mathrm{C}\right)$ for 45 minutes. The reaction was stopped by the addition of electrophoresis sample buffer and heating at $95^{\circ} \mathrm{C}$ for 3 minutes. The samples were then separated by SDS-PAGE for $2.5 \mathrm{hrs}$ at $12 \mathrm{~mA}$, silver stained, dried, and subsequently exposed to X-ray film for visualization.

2.10. Statistics. A mixed model incorporating linear regression and analysis of covariance was used to compare response variable (stroke volume) slopes plotted versus end diastolic volume, using treatment (baseline versus dobutamine) as the independent variable. Slopes of length-tension relationships were determined by linear regression. Paired $t$ tests were used to determine whether there were significant differences in length-tension slopes and force-velocity parameters at two different sarcomere lengths or before and after PKA treatments. $P<0.05$ was chosen as indicating significance. All values are expressed as means \pm SD unless, otherwise, noted.

\section{Results}

3.1. Sarcomere Length Dependence of Force. The characteristics of porcine left ventricular cardiac myocyte preparations 

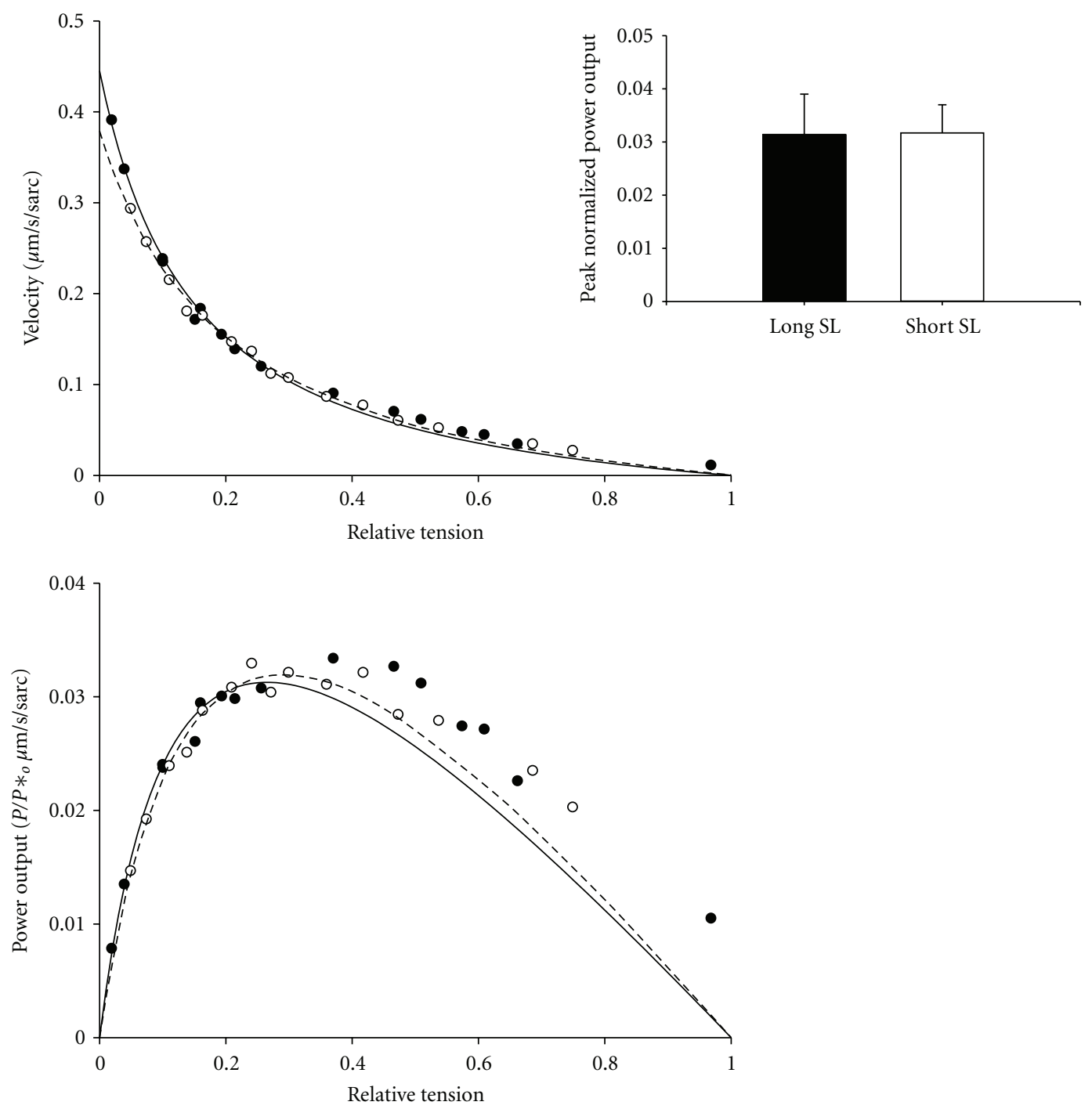

- Sarcomere length $2.3 \mu \mathrm{m}$

○ Sarcomere length $1.9 \mu \mathrm{m}$

FIGURE 4: Normalized force-velocity and power-load curves from a pig left ventricular myocyte preparation at long and short sarcomere length obtained during half-maximal $\mathrm{Ca}^{2+}$ activations. Pig cardiac myocyte preparations exhibited little sarcomere length dependence of loaded shortening and power output. Inset shows bar plot (mean \pm SD) of peak normalized power output at long $(\sim 2.30 \mu \mathrm{m})$ and short $(\sim 1.90 \mu \mathrm{m})$ sarcomere length ( $n=8$ myocyte preparations).

are provided in Table 1. Steady-state sarcomere length-tension relationships were examined in myocyte preparations during near-half-maximal $\mathrm{Ca}^{2+}$ activations. Interestingly, porcine cardiac myocyte preparations exhibited a dichotomy of sarcomere length-tension relationships, some had shallow sarcomere length-tension relations while others displayed steep relationships (Figure 1). Histogram analysis of the length-tension relationship slopes indicates near bimodal distribution with one population of cells having a slope near 1.0 and another population with a slope near 1.5 (Figure 1), which has been similarly reported in rat and ferret myocyte preparations [22, 31]. We next examined whether PKAinduced phosphorylation of myofilament proteins may mediate the distribution of length-tension populations. PKA shifted a shallow length tension relationship to a steep length tension relationship implicating phosphorylation of myosin binding protein-C (MyBP-C) and/or cardiac troponin I (cTnI) as molecular modulators of sarcomere length-tension curves in porcine cardiac myocytes (Figure 2), as was previously observed in rat cardiac myocyte preparations [22].

3.2. Sarcomere Length Dependence of Rates of Force Development $\left(k_{\mathrm{tr}}\right)$. The rate of force development is thought to mediate pressure development rates in mammalian ventricles. We examined the sarcomere length-dependence of force development rates in porcine cardiac myocytes. Force redevelopment was measured after a slack re-stretch maneuver, and the rate constant of force development $\left(k_{\mathrm{tr}}\right)$ was 


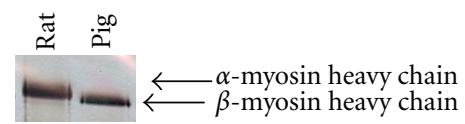

(a)

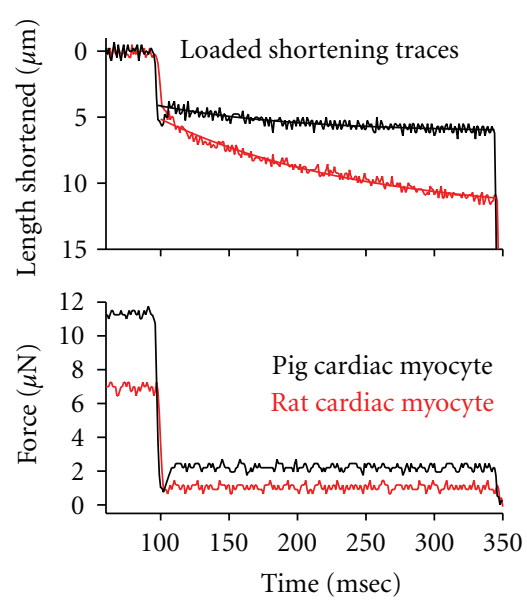

(b)

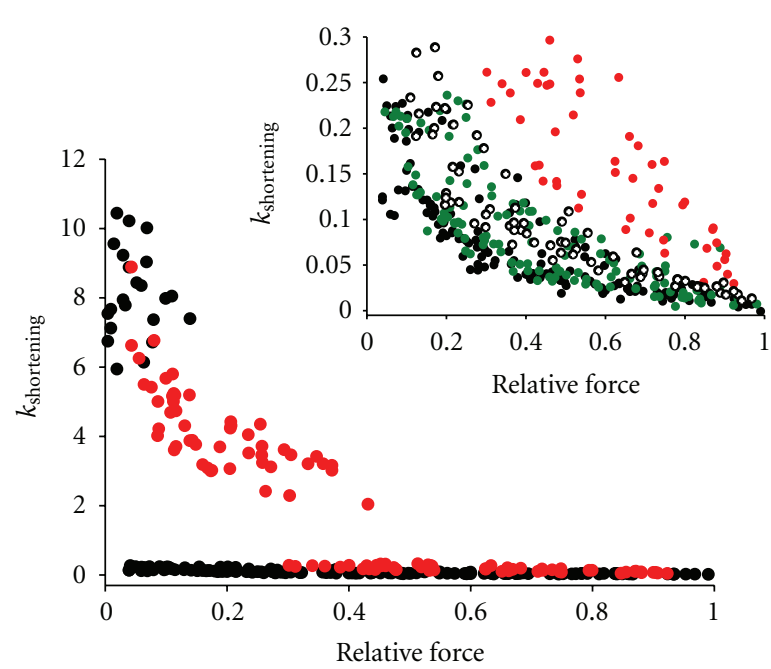

- Rat myocytes

- Pig myocytes

- Pig myocytes with steep L-TR

- PKA treated pig myocytes

(c)

FIGURE 5: (a) Silver-stained gel showing the myosin heavy chain isoforms contained in a rat cardiac myocyte preparation compared to a pig cardiac myocyte preparation. (b) Representative length and force traces during a lightly loaded force clamp in a rat cardiac myocyte preparation (red) and a pig cardiac myocyte preparation (black) during a submaximal $\mathrm{Ca}^{2+}$ activation. (c) Length traces exhibited considerably greater curvature (greater $k_{\text {shortening }}$ ) in rat myocytes compared to pig myocyte preparations at all relative loads. Inset in C shows

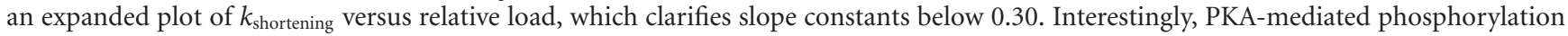
increased the curvature $\left(k_{\text {shortening }}\right)$ of length traces towards those of rat myocyte preparations. In addition, pig cardiac myocyte preparations that exhibited steep length-tension relationships (L-T R) also had more curved length traces. This is consistent with PKA-mediated phosphorylation of myofilaments yielding greater responsiveness to changes in sarcomere length, in this case exhibited by greater shorteninginduced cooperative deactivation.

calculated by fitting a single concave exponential equation to the force trace. At sarcomere length $\sim 2.30 \mu \mathrm{m}, k_{\mathrm{tr}}$ was $\sim 0.3 \mathrm{~s}^{-1}$ during half-maximal activation, which was similar to previously reported for pig myocytes [32], nearly an order of magnitude lower than that measured in rat cardiac myocyte preparations, and only $30 \%$ of $k_{\text {tr }}$ values in rat slow-twitch skeletal muscle fibers, which like porcine cardiac myocytes contain the $\beta$-myosin heavy chain isoform. As sarcomere length was reduced from $\sim 2.30 \mu \mathrm{m}$ to $1.90 \mu \mathrm{m}, k_{\mathrm{tr}}$ remained relatively constant, and then at sarcomere lengths below $1.90 \mu \mathrm{m}, k_{\text {tr }}$ progressively increased. This $k_{\text {tr }}-\mathrm{SL}$ relationship was qualitatively similar to that observed in rat slow-twitch skeletal muscle fibers (Figure 3). Since force falls as sarcomere length is decreased but $k_{\mathrm{tr}}$ increased with shorter sarcomere lengths, this implicates that sarcomere length per se can override the well-described $\mathrm{Ca}^{2+}$-activated force dependence of rates of force redevelopment in cardiac muscle [33-35], that is, sarcomere length plays a dominant role in the kinetics of myofibrillar mechanical properties.

3.3. Sarcomere Length Dependence of Force-Velocity and Power-Load Curves. Previous work has shown a tight regulation between isometric force and normalized force-velocity relationships in rat-skinned cardiac myocyte preparations [26]. However, in porcine cardiac myocyte preparations there was no force dependence of normalized force-velocity and power-load curves when force was altered by changing sarcomere length (i.e., force fell $\sim 50 \%$ when sarcomere length was shortened from $\sim 2.30 \mu \mathrm{m}$ to $1.90 \mu \mathrm{m}$ at the same submaximal activator $\left[\mathrm{Ca}^{2+}\right]$, Figure 4$)$. The finding that normalized myocyte power did not change over this sarcomere length range in pig myocytes differs from rat cardiac myocyte preparations where normalized force-velocity relationships were shifted downward at short sarcomere length (i.e., $\sim 1.90 \mu \mathrm{m}$ versus $\sim 2.30 \mu \mathrm{m})$ at the same activator $\left[\mathrm{Ca}^{2+}\right]$ [36]. The reason for this species difference is not known. One possibility is differences in cardiac myosin heavy chain; rat myocytes contain predominantly $\alpha$-MyHC while pig myocytes contain mostly $\beta$-MyHC (Figure 5(a)). Interestingly, porcine $\beta$-MyHC has been shown to have a very slow actin-activated ATPase activity [37], which would prolong the duty cycle (i.e., cross-bridge cycle time spent strongly attached to actin). These strongly attached cross-bridges would tend to keep the thin filament activated [38-40] 

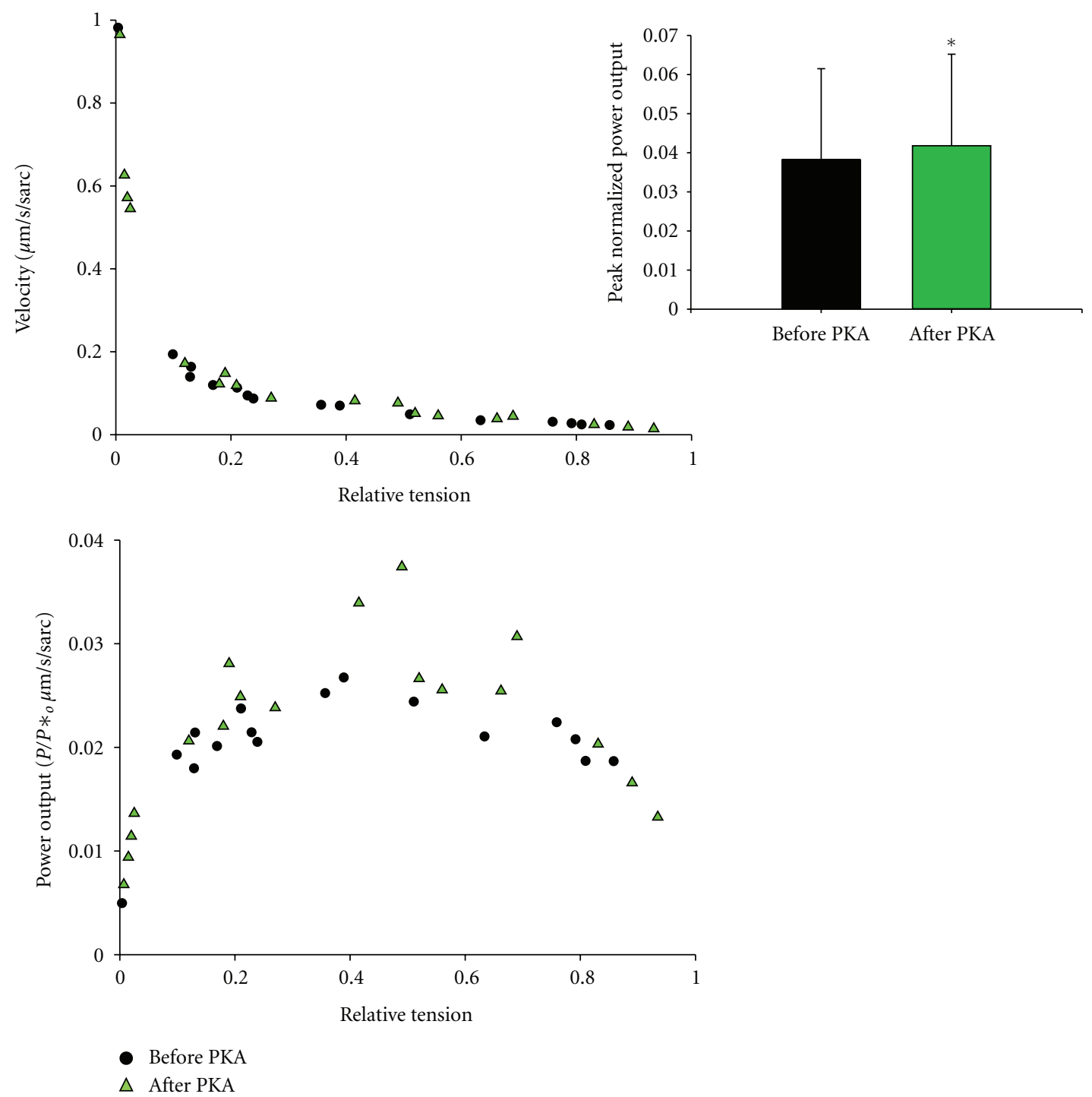

FIGURE 6: Normalized force-velocity and power-load curves from a pig left ventricular myocyte preparation before and after PKA treatment during half-maximal $\mathrm{Ca}^{2+}$ activations. Pig cardiac myocyte preparations exhibited more power after PKA treatment. Inset shows bar plot $($ mean $\pm \mathrm{SD})$ of peak normalized power output before and after PKA ( $n=10$ myocyte preparations).

throughout the duration of the force clamp. This would sustain a relatively high number of cross-bridges to work against the load(s). This is consistent with linear length traces during load clamps in pig cardiac myocyte preparations (Figure 5(b)). The extent of curvature of length traces during load clamps is quantified by $k_{\text {shortening }}$ values, which were much lower in pig myocytes than rat myocytes (Figure 5(c)). In pig myocytes, length traces were nearly linear (as indexed

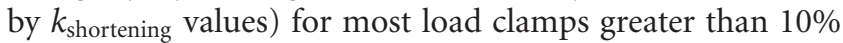
isometric force. This differs markedly from rat cardiac myocyte preparations, whereby length traces during force clamps deviate from linear at load clamps near $40 \%$ isometric force during submaximal $\mathrm{Ca}^{2+}$ activations (Figure 5(c)).

3.4. PKA Effects on Loaded Shortening, Power Output, and $k_{\text {shortening. }}$. We have previously shown that peak power generating capacity increases after PKA-mediated phosphorylation in rat cardiac myocyte preparations $[25,30]$ and such a change may contribute to augmented contractility in working hearts (i.e., more stroke volume at a given enddiastolic volume) [12]. We examined if a similar biophysical response would occur in pig cardiac myocyte preparations as a potential means for physiological changes in ventricular contractility in response to $\beta$-adrenergic stimulation and its downstream signaling molecule, protein kinase A (PKA). We observed a statistically significant increase in peak normalized power output after PKA treatment in pig myocyte preparations (Figure 6), however, the increase was considerably smaller than observed in rat myocyte preparations (a $10 \%$ increase in pig myocytes versus a 33\% increase in rat myocytes $[25,30])$. This small increase in myocyte power after PKA is consistent with a relatively small leftward shift 
in ventricular function illustrated by an $\sim 15 \%$ increase in stroke volume for a given end diastolic volume that we observed in anesthetized pigs in response to a $5 \mu \mathrm{g} / \mathrm{kg} / \mathrm{min}$ dose of dobutamine at a mean arterial pressure of $80 \mathrm{mmHg}$ (Figure 7).

Interestingly, PKA-mediated phosphorylation increased the curvature ( $k_{\text {shortening }}$ ) of length traces towards those of rat myocyte preparations (Figure 5(c)). In addition, pig cardiac myocyte preparations that exhibited steep length-tension relationships also had more curved length traces. This is consistent with the idea that PKA-mediated phosphorylation of myofilaments yields both greater force responsiveness to sarcomere length and greater shortening-induced cooperative deactivation.

In summary, pig cardiac myocyte preparations showed two populations of sarcomere length-tension relationships, which appear to be modulated by PKA. Sarcomere length overrode the $\mathrm{Ca}^{2+}$-activated-force dependence of $k_{\text {tr }}$ and loaded shortening. PKA treatment also slightly sped loaded shortening especially at loads optimal for power and yielded more curvilinear length traces during force clamps.

\section{Discussion}

In order to better understand the intricacies of heart function, it is necessary to determine the intermolecular control of myofibrillar contraction. In this study, we focused on three key myofibrillar functional properties (i) force, (ii) rate of force development, and (iii) power generating capacity, which together dictate ventricular stroke volume. We systematically examined these properties in porcine myofibrillar preparations. The study used pig ventricular myocardium for two main reasons: (1) pig hearts have many similarities to human hearts including heart size, heart rate, coronary circulation, responsiveness to many pharmacologic agents, and expression of mostly -myosin heavy chain (MyHC), and (2) to make comparisons with rat myocardium, which have been more extensively studied $[7,8,12,22,25,26,30,36$, 41]. Overall, pigs likely provide an advantageous model to study cellular mechanisms of ventricular function and provide further basic insight into potential defects in cardiomyopathic states more related to the human condition.

We observed that sarcomere-length dependence of force in pig myocyte preparations was very similar to that previously observed in rat cardiac myocyte preparations [22]. There was a dichotomy in the steepness of sarcomere length tension relationship whereby one population was shallower than the other. Interestingly, when myocyte preparations with a shallow length-tension relationship were treated with PKA, the relationships became steeper. While the exact molecular (posttranslational) modification by which PKA steepens length tension relationships remains to be determined, the finding is consistent with steeper ventricular function curves in response to $\beta$-adrenergic stimulation, assuming that myocyte length-tension contributes, at least in part, to the cellular basis of the Frank-Starling relationship. PKA also increased loaded shortening especially at loads near peak power and increased the curvature of length traces during

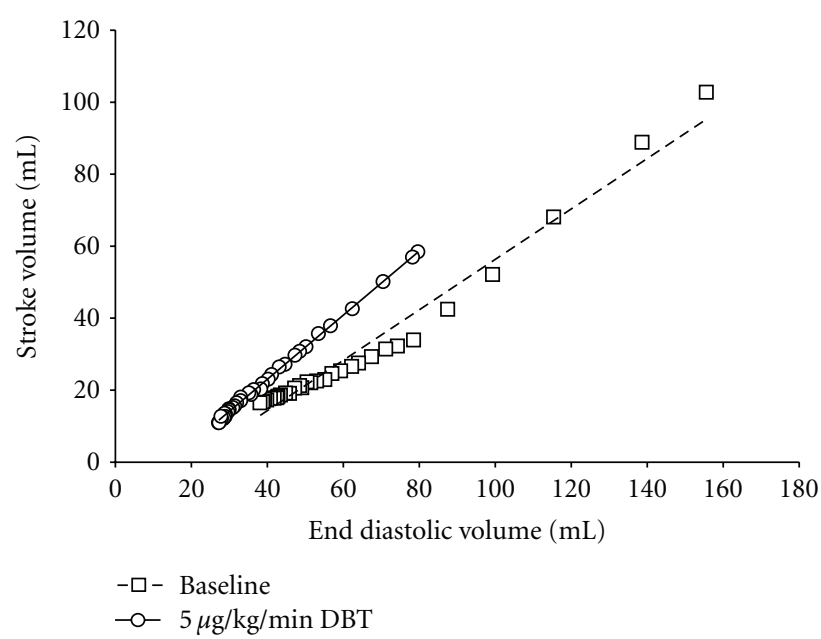

(a)

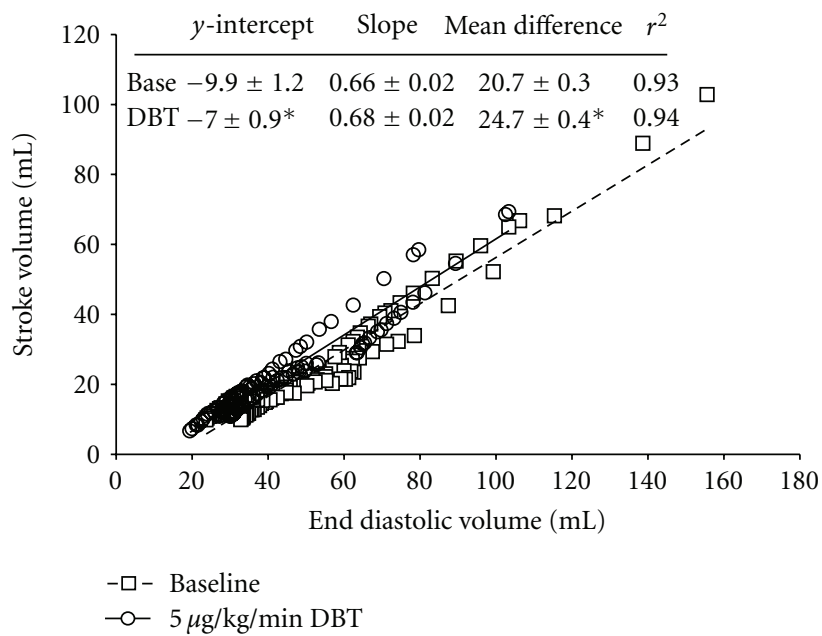

(b)

Figure 7: (a) Representative Frank-Starling relationship from one animal at baseline (Base) and after treatment with dobutamine (DBT). (b) Comprehensive group data from all animals illustrating a significant leftward shift in the Frank-Starling relationship (mixed model, treatment main effect adjusted for EDV covariance, $P<$ $0.05)$. There was no significant interaction or change in slope of the Frank-Starling relationship between treatments (see table inset in (b)), therefore, parallelism was assumed. The $y$-intercept and marginal mean difference were both significantly increased following the dobutamine treatment $\left({ }^{*} P<0.05\right.$; table inset (b)). The dobutamine challenge resulted in a $\sim 15 \%$ increase in stroke volume (SV) for a given end diastolic volume (EDV) in vivo. This increase in ventricular function was similar in magnitude to that observed in our myocyte preparations $(\sim 10 \%)$, illustrating the coherence of our whole heart and cardiac myocyte functional data.

force clamps. These PKA-mediated changes in myofibrillar function are consistent with physiological changes induced by $\beta$-adrenergic stimulation. $\beta$-adrenergic stimulation is known to (i) increase contractility (mediated in part by greater myocyte power at a given sarcomere length), (ii) steepen the Frank-Starling relationship (mediated in part by 


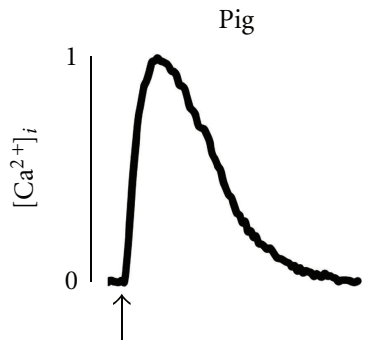

(a)

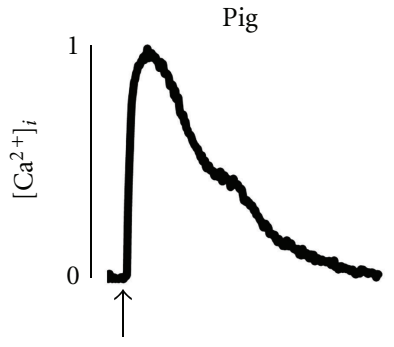

(b)

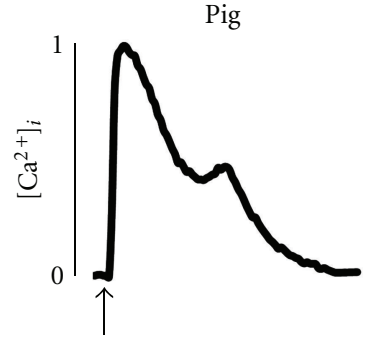

(c)

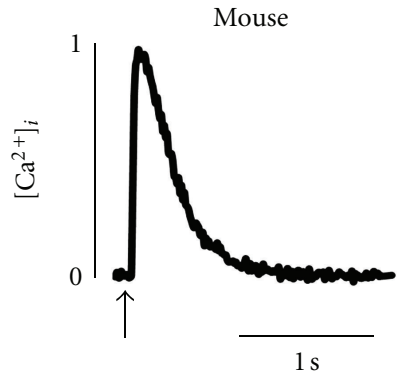

(d)

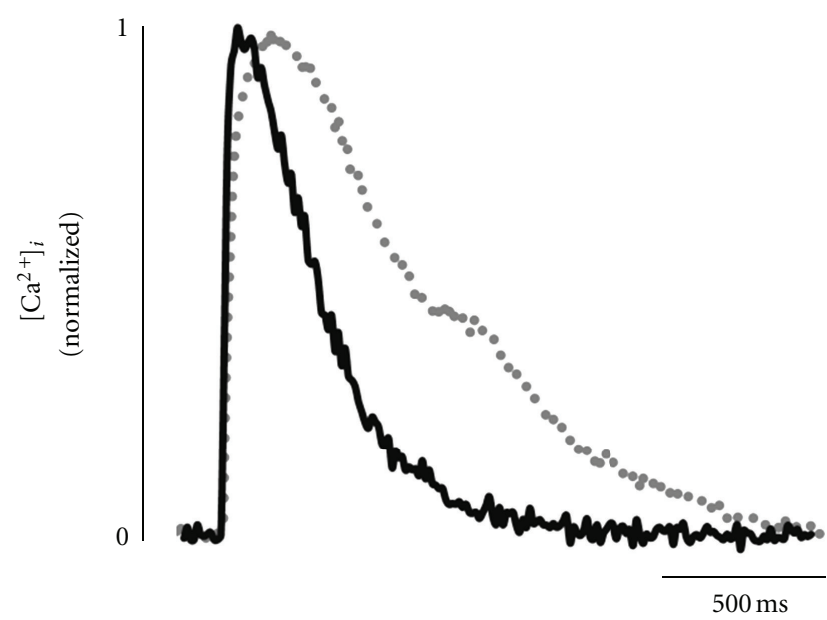

(e)

Figure 8: Representative amplitude-normalized calcium transients of Pig ((a)-(c)) and Mouse (d) left-ventricular myocytes (0.5 Hz, field stimulus denoted by arrow). Calcium transients from Pig exhibited multiple waveforms, including normal recovery from the transient peak (a, 2 of 14 cells), recovery with a marked shoulder ((b), 8 of 14 cells), and recovery with a secondary increase in calcium (c, 4 of 14 cells). (d) Mouse transients consistently exhibited a rapid transient recovery $(n=40)$. (e) Overlay of transients shown in (b) and (d) illustrating the distinct transient kinetics between pig (gray) and mouse (black) myocytes.

steeper length dependence of force), and (iii) speed relaxation (mediated, in part, by greater extent of shorteninginduced cooperative deactivation manifested by more curved length traces). Interestingly, these myofibrillar changes in pig myocytes were all quantitatively less than those observed in rat cardiac myocytes, which is consistent with a slightly lower cardiac reserve that we have observed in pig hearts compared to rat and human hearts $[42,43]$.

Additional myofibrillar mechanical properties observed in pig myocytes were that at the same activator $\left[\mathrm{Ca}^{2+}\right]$ there was limited sarcomere length dependence of $k_{\mathrm{tr}}$ and forcevelocity relationships. The sarcomere length dependence of $k_{\text {tr }}$ was similar to that observed in rat slow-twitch skeletal muscle fibers in which $k_{\text {tr }}$ was similar over sarcomere length range of $\sim 2.30$ to $1.90 \mathrm{um}$ and then increased at shorter sarcomere lengths. Since force falls over this entire sarcomere length range, this indicates that sarcomere length overrides the force dependence of $k_{\text {tr }}$ previously reported in cardiac muscle [33-35]. The mechanistic reasons for sarcomere length dominance of $k_{\text {tr }}$ is unclear but may indicate that cooperative activation of thin filaments is progressively reduced at shorter sarcomere lengths perhaps by more compliant thin filaments (i.e., shorter persistence length, which is the length that a mechanical force is transmitted along a functional entity), which would result in less recruitment of cross-bridges from the noncycling pool into the cycling pool, which has been proposed to limit rate of force development $[35,44]$. Conversely, the lack of sarcomere length dependence of loaded shortening and power in pig myocytes differs from rat cardiac myocytes, where power decreased at short sarcomere length at the same activator $\left[\mathrm{Ca}^{2+}\right][36]$. This may arise due to the very slow actin-activated ATPase activity reported for porcine $\beta$-MyHC [37]. Slow crossbridge detachment would increase the population of strongly bound cross-bridges, which are thought to shift the thin filament equilibrium towards the open state by direct interaction with the actin-tropomyosin interface [40] and, at least in cardiac muscle, by increased affinity of cTnC for $\mathrm{Ca}^{2+}[45$, 46]. Interestingly, we observed a marked shoulder in $\mathrm{Ca}^{2+}$ transients from intact pig myocytes (Figure 8). This shoulder was not observed in mouse myocytes that contain $\alpha$-MyHC, which has a relatively short duty cycle. Mechanistically, the $\mathrm{Ca}^{2+}$ transient shoulder may arise from delayed $\mathrm{Ca}^{2+}$ dissociation from cTnC due to prolonged strongly bound attachment state(s) inherent to the long duty cycle of $\beta$ MyHC cross-bridges expressed in pig cardiac myocytes. 
Additionally, significantly delayed dissociation of $\mathrm{Ca}^{2+}$ from cTnC may elevate cytosolic $\mathrm{Ca}^{2+}$ at a time when ryanodine receptors have recovered from inactivation, yielding secondary $\mathrm{Ca}^{2+}$ induced- $\mathrm{Ca}^{2+}$ release from the sarcoplasmic reticulum. It does, however, appear that the long duty cycle of pig $\beta$-MyHC can be modulated by PKA mediated phosphorylation of myofibrillar proteins since PKA elevated power and yielded more curved length traces during load clamps (i.e., greater $k_{\text {shortening }}$ values). These PKA mediated effects appear qualitatively similar across species and are thus likely to be of important physiological significance. In terms of ventricular contraction, PKA tends to increase the cooperative activation of thin filaments [47-49] providing more force-generating cross-bridges to work against an afterload yielding faster loaded shortening and, thus, more ejection. In terms of relaxation, the greater curvature of length traces implies greater shortening-induced cooperative deactivation, which would assist in ventricular relaxation to allow more time for filling and, thus, preserve stroke volume in the midst of higher heart rates. This mechanical signaling paradigm provides a myofibrillar mechanism to optimize cardiac output in response to high peripheral demands associated with stress (e.g., exercise).

\section{Acknowledgments}

This work is supported by a National Heart, Lung, and Blood Institute grant (R01-HL-57852) to K. S. M., and American Heart Association (Heartland Affiliate) Postdoctoral Fellowship (0825725G) to L. M. H., an NIH grant (HL52490) to D. K. Bowles, an NIH grant (HL R01-HL086483) to S. S. Segal, and an NIH grant (HL09398-01) to C. A. E.

\section{References}

[1] A. Fabiato and F. Fabiato, "Dependence of the contractile activation of skinned cardiac cells on the sarcomere length," Nature, vol. 256, no. 5512, pp. 54-56, 1975.

[2] G. H. Rossmanith, J. F. Y. Hoh, A. Kirman, and L. J. Kwan, "Influence of V1 and V3 isomyosins on the mechanical behaviour of rat papillary muscle as studied by pseudo-random binary noise modulated length perturbations," Journal of Muscle Research and Cell Motility, vol. 7, no. 4, pp. 307-319, 1986.

[3] P. P. De Tombe and H. E. D. J. Ter Keurs, "Force and velocity of sarcomere shortening in trabeculae from rat heart. Effects of temperature," Circulation Research, vol. 66, no. 5, pp. 12391254, 1990.

[4] N. K. Sweitzer and R. L. Moss, "The effect of altered temperature on $\mathrm{Ca}^{2+}$-sensitive force in permeabilized myocardium and skeletal muscle. Evidence for force dependence of thin filament activation," Journal of General Physiology, vol. 96, no. 6, pp. 1221-1245, 1990.

[5] M. Puceat, O. Clement, P. Lechene, J. M. Pelosin, R. VenturaClapier, and G. Vassort, "Neurohormonal control of calcium sensitivity of myofilaments in rat single heart cells," Circulation Research, vol. 67, no. 2, pp. 517-524, 1990.

[6] K. T. Strang, N. K. Sweitzer, M. L. Greaser, and R. L. Moss, " $\beta$-Adrenergic receptor stimulation increases unloaded shortening velocity of skinned single ventricular myocytes from rats," Circulation Research, vol. 74, no. 3, pp. 542-549, 1994.
[7] K. S. McDonald, M. R. Wolff, and R. L. Moss, "Force-velocity and power-load curves in rat skinned cardiac myocytes," Journal of Physiology, vol. 511, no. 2, pp. 519-531, 1998.

[8] D. P. Fitzsimons, J. R. Patel, and R. L. Moss, "Cross-bridge interaction kinetics in rat myocardium are accelerated by strong binding of myosin to the thin filament," Journal of Physiology, vol. 530, no. 2, pp. 263-272, 2001.

[9] O. Cazorla, Y. Wu, T. C. Irving, and H. Granzier, "Titin-based modulation of calcium sensitivity of active tension in mouse skinned cardiac myocytes," Circulation Research, vol. 88, no. 10, pp. 1028-1035, 2001.

[10] M. Regnier, H. Martin, R. J. Barsotti, A. J. Rivera, D. A. Martyn, and E. Clemmens, "Cross-bridge versus thin filament contributions to the level and rate of force development in cardiac muscle," Biophysical Journal, vol. 87, no. 3, pp. 1815-1824, 2004.

[11] P. P. Chen, J. R. Patel, I. N. Rybakova, J. W. Walker, and R. L. Moss, "Protein kinase A-induced myofilament desensitization to $\mathrm{Ca}^{2+}$ as a result of phosphorylation of cardiac myosin-binding protein C," Journal of General Physiology, vol. 136, no. 6, pp. 615-627, 2010.

[12] F. S. Korte, T. J. Herron, M. J. Rovetto, and K. S. McDonald, "Power output is linearly related to MyHC content in rat skinned myocytes and isolated working hearts," American Journal of Physiology, Heart and Circulatory Physiology, vol. 289, no. 2, pp. H801-H812, 2005.

[13] R. J. Belin, M. P. Sumandea, E. J. Allen et al., "Augmented protein kinase $\mathrm{C}$ - $\alpha$-induced myofilament protein phosphorylation contributes to myofilament dysfunction in experimental congestive heart failure," Circulation Research, vol. 101, no. 2, pp. 195-204, 2007.

[14] G. Nowak, J. R. Peña, D. Urboniene, D. L. Geenen, R. J. Solaro, and B. M. Wolska, "Correlations between alterations in length-dependent $\mathrm{Ca}^{2+}$ activation of cardiac myofilaments and the end-systolic pressure-volume relation," Journal of Muscle Research and Cell Motility, vol. 28, no. 7-8, pp. 415-419, 2007.

[15] K. S. McDonald, P. P. A. Mammen, K. T. Strang, R. L. Moss, and W. P. Miller, "Isometric and dynamic contractile properties of porcine skinned cardiac myocytes after stunning," Circulation Research, vol. 77, no. 5, pp. 964-972, 1995.

[16] D. J. Duncker, N. M. Boontje, D. Merkus et al., "Prevention of myofilament dysfunction by $\beta$-blocker therapy in postinfarct remodeling," Circulation: Heart Failure, vol. 2, no. 3, pp. 233242, 2009.

[17] J. E. Stelzer, H. S. Norman, P. P. Chen, J. R. Patel, and R. L. Moss, "Transmural variation in myosin heavy chain isoform expression modulates the timing of myocardial force generation in porcine left ventricle," Journal of Physiology, vol. 586, no. 21, pp. 5203-5214, 2008.

[18] C. A. Emter and C. P. Baines, "Low-intensity aerobic interval training attenuates pathological left ventricular remodeling and mitochondrial dysfunction in aortic-banded miniature swine," American Journal of Physiology, Heart and Circulatory Physiology, vol. 299, no. 5, pp. H1348-H1356, 2010.

[19] A. C. Hinken, F. S. Korte, and K. S. McDonald, "Porcine cardiac myocyte power output is increased after chronic exercise training," Journal of Applied Physiology, vol. 101, no. 1, pp. 4046, 2006.

[20] A. Fabiato, "Computer programs for calculating total from specified free or free from specified total ionic concentrations in aqueous solutions containing multiple metals and ligands," Methods in Enzymology, vol. 157, no. C, pp. 378-417, 1988. 
[21] R. L. Moss, "Sarcomere length-tension relations of frog skinned muscle fibres during calcium activation at short lengths," Journal of Physiology, vol. 292, pp. 177-192, 1979.

[22] L. M. Hanft and K. S. McDonald, "Length dependence of force generation exhibit similarities between rat cardiac myocytes and skeletal muscle fibres," The Journal of Physiology, vol. 588, no. 15, pp. 2891-2903, 2010.

[23] F. S. Korte, K. S. McDonald, S. P. Harris, and R. L. Moss, "Loaded shortening, power output, and rate of force redevelopment are increased with knockout of cardiac myosin binding protein-C," Circulation Research, vol. 93, no. 8, pp. 752758, 2003.

[24] A. C. Hinken and K. S. McDonald, "Inorganic phosphate speeds loaded shortening in rat skinned cardiac myocytes," American Journal of Physiology, Cell Physiology, vol. 287, no. 2, pp. C500-C507, 2004.

[25] L. M. Hanft and K. S. McDonald, "Sarcomere length dependence of power output is increased after PKA treatment in rat cardiac myocytes," American Journal of Physiology, Heart and Circulatory Physiology, vol. 296, no. 5, pp. H1524-H1531, 2009.

[26] K. S. McDonald, " $\mathrm{Ca}^{2+}$ dependence of loaded shortening in rat skinned cardiac myocytes and skeletal muscle fibres," Journal of Physiology, vol. 525, no. 1, pp. 169-181, 2000.

[27] A. V. Hill, "The heat of shortening and the dynamic constants of muscle," Proceedings of the Royal Society B, vol. 126, pp. 136$195,1938$.

[28] R. C. Woledge, N. A. Curtin, and E. Homsher, Energetic Aspects of Muscle Contraction, Academic Press, London, UK, 1985.

[29] J. M. Metzger, P. A. Wahr, D. E. Michele, F. Albayya, and M. V. Westfall, "Effects of myosin heavy chain isoform switching on $\mathrm{Ca}^{2+}$-activated tension development in single adult cardiac myocytes," Circulation Research, vol. 84, no. 11, pp. 13101317, 1999.

[30] T. J. Herron, F. S. Korte, and K. S. McDonald, "Power output is increased after phosphorylation of myofibrillar proteins in rat skinned cardiac myocytes," Circulation Research, vol. 89, no. 12, pp. 1184-1190, 2001.

[31] O. Cazorla, J. Y. Le Guennec, and E. White, "Length-Tension relationships of sub-epicardial and sub-endocardial single ventricular myocytes from rat and ferret hearts," Journal of Molecular and Cellular Cardiology, vol. 32, no. 5, pp. 735-744, 2000.

[32] I. F. Édes, D. Czuriga, G. Csányi et al., "Rate of tension redevelopment is not modulated by sarcomere length in permeabilized human, murine, and porcine cardiomyocytes," American Journal of Physiology, Regulatory Integrative and Comparative Physiology, vol. 293, no. 1, pp. R20-R29, 2007.

[33] M. R. Wolff, K. S. McDonald, and R. L. Moss, "Rate of tension development in cardiac muscle varies with level of activator calcium," Circulation Research, vol. 76, no. 1, pp. 154-160, 1995.

[34] C. Vannier, H. Chevassus, and G. Vassort, "Ca-dependence of isometric force kinetics in single skinned ventricular cardiomyocytes from rats," Cardiovascular Research, vol. 32, no. 3, pp. 580-586, 1996.

[35] D. P. Fitzsimons, J. R. Patel, K. S. Campbell, and R. L. Moss, "Cooperative mechanisms in the activation dependence of the rate of force development in rabbit skinned skeletal muscle fibers," Journal of General Physiology, vol. 117, no. 2, pp. 133$148,2001$.

[36] F. S. Korte and K. S. Mcdonald, "Sarcomere length dependence of rat skinned cardiac myocyte mechanical properties: dependence on myosin heavy chain," Journal of Physiology, vol. 581, no. 2, pp. 725-739, 2007.

[37] J. S. Pereira, D. Pavlov, M. Nili, M. Greaser, E. Homsher, and R. L. Moss, "Kinetic differences in cardiac myosins with identical loop 1 sequences," Journal of Biological Chemistry, vol. 276, no. 6, pp. 4409-4415, 2001.

[38] D. F. A. McKillop and M. A. Geeves, "Regulation of the interaction between actin and myosin subfragment 1: evidence for three states of the thin filament," Biophysical Journal, vol. 65, no. 2, pp. 693-701, 1993.

[39] P. Vibert, R. Craig, and W. Lehman, "Steric-model for activation of muscle thin filaments," Journal of Molecular Biology, vol. 266, no. 1, pp. 8-14, 1997.

[40] W. Lehman, A. Galińska-Rakoczy, V. Hatch, L. S. Tobacman, and R. Craig, "Structural basis for the activation of muscle contraction by troponin and tropomyosin," Journal of Molecular Biology, vol. 388, no. 4, pp. 673-681, 2009.

[41] G. M. Diffee and E. Chung, "Altered single cell force-velocity and power properties in exercise-trained rat myocardium," Journal of Applied Physiology, vol. 94, no. 5, pp. 1941-1948, 2003.

[42] E. Plante, D. Lachance, M. C. Drolet, E. Roussel, J. Couet, and M. Arsenault, "Dobutamine stress echocardiography in healthy adult male rats," Cardiovascular Ultrasound, vol. 3, article no. 34, 2005.

[43] H. S. Norman, J. Oujiri, S. J. Larue, C. B. Chapman, K. B. Margulies, and N. K. Sweitzer, "Decreased cardiac functional reserve in heart failure with preserved systolic function," Journal of Cardiac Failure, vol. 17, no. 4, pp. 301-308, 2011.

[44] K. Campbell, "Rate constant of muscle force redevelopment reflects cooperative activation as well as cross-bridge kinetics," Biophysical Journal, vol. 72, no. 1, pp. 254-262, 1997.

[45] P. A. Hofmann and F. Fuchs, "Evidence for a force-dependent component of calcium binding to cardiac troponin C," American Journal of Physiology, vol. 253, pp. C541-C546, 1987.

[46] P. A. Hofmann and F. Fuchs, "Effect of length and cross-bridge attachment on $\mathrm{Ca}^{2+}$ binding to cardiac troponin C," American Journal of Physiology, vol. 253, no. 1, pp. C90-C96, 1987.

[47] J. Van Der Velden, J. W. De Jong, V. J. Owen, P. B. J. Burton, and G. J. M. Stienen, "Effect of protein kinase A on calcium sensitivity of force and its sarcomere length dependence in human cardiomyocytes," Cardiovascular Research, vol. 46, no. 3, pp. 487-495, 2000.

[48] J. P. Konhilas, T. C. Irving, B. M. Wolska et al., "Troponin I in the murine myocardium: influence on length-dependent activation and interfilament spacing," Journal of Physiology, vol. 547, no. 3, pp. 951-961, 2003.

[49] L. M. Hanft and K. S. McDonald, "Determinants of loaded shortening in rat cardiac myocytes 2010," Biophysical Society Meetings. In press. 

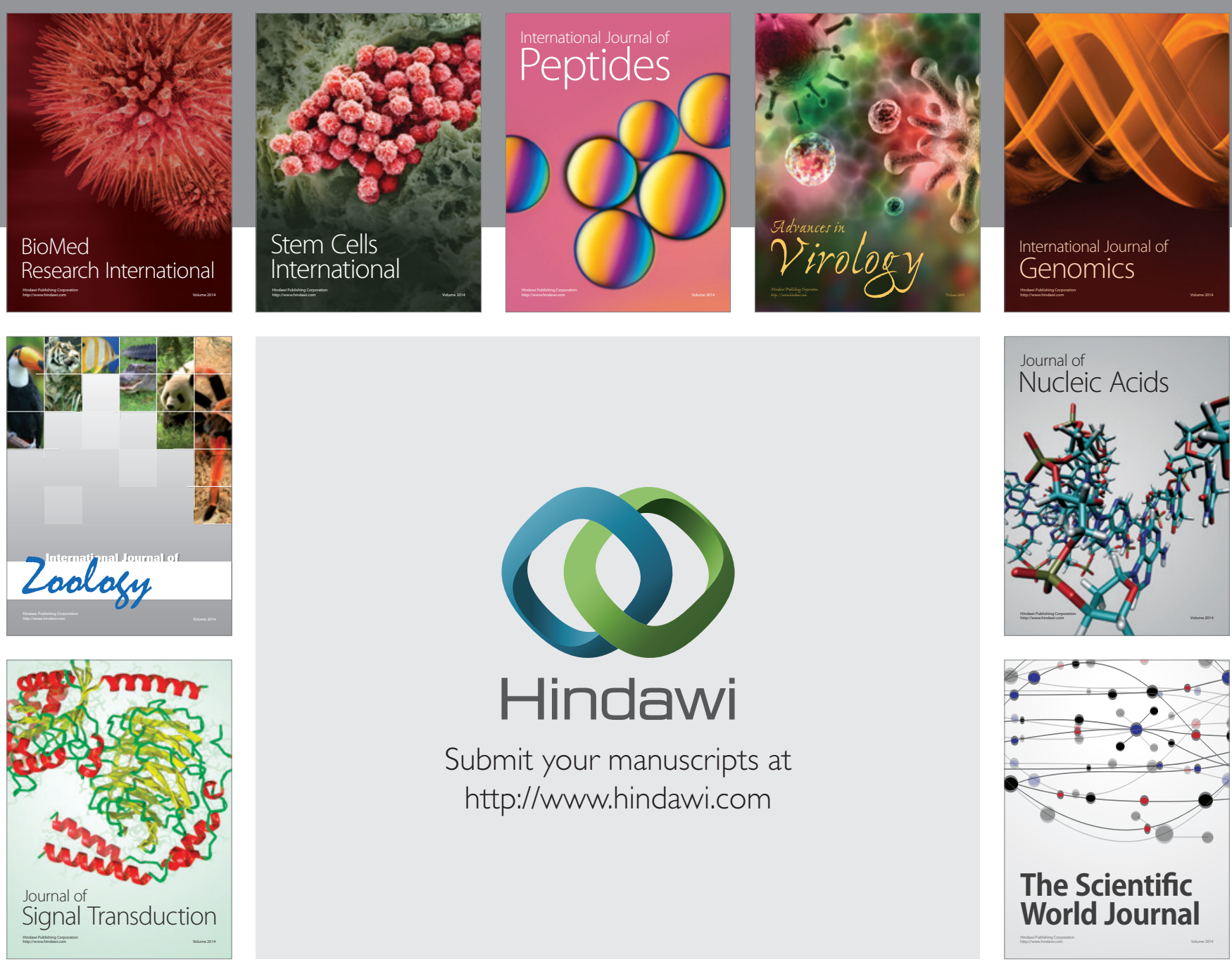

Submit your manuscripts at

http://www.hindawi.com
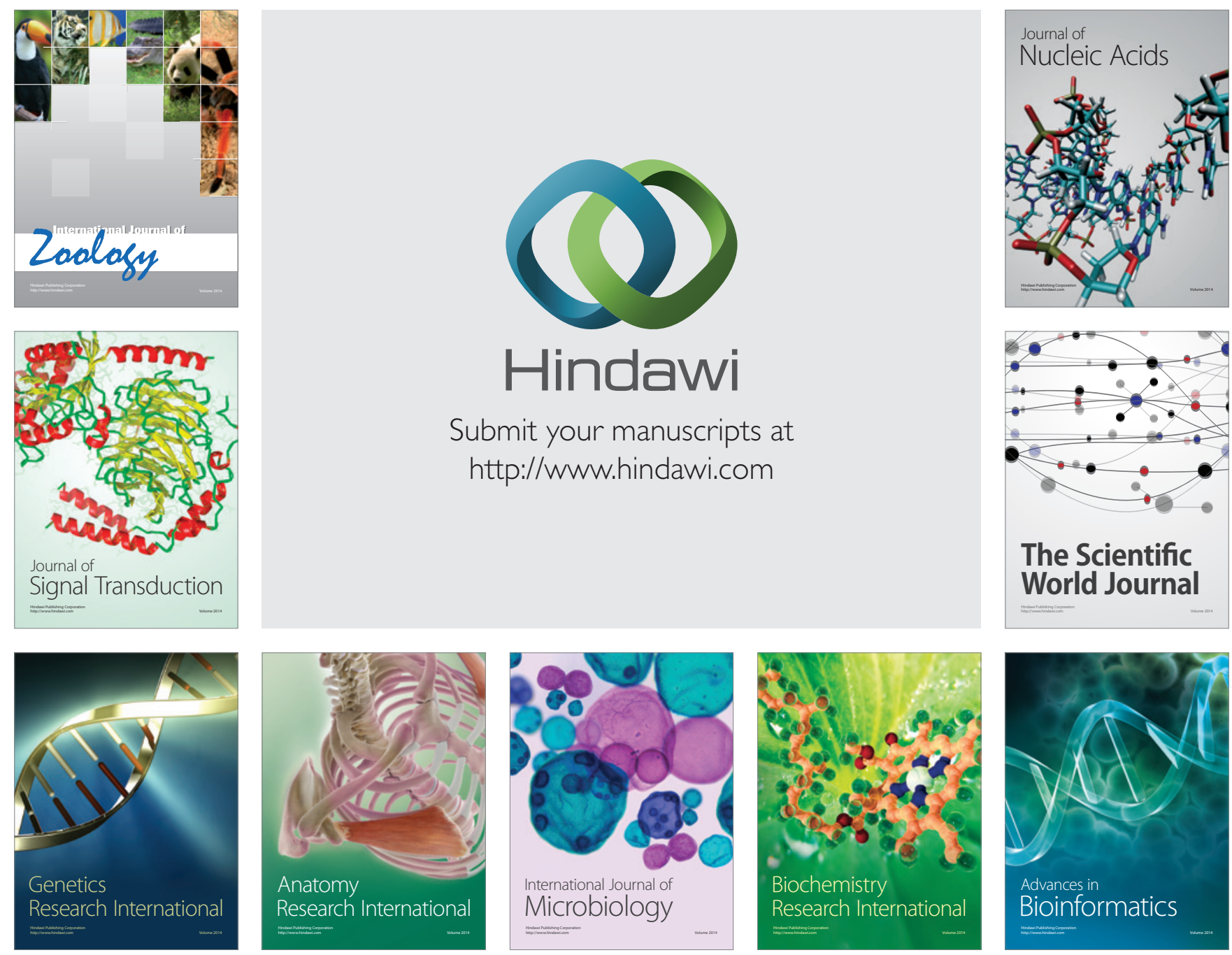

The Scientific World Journal
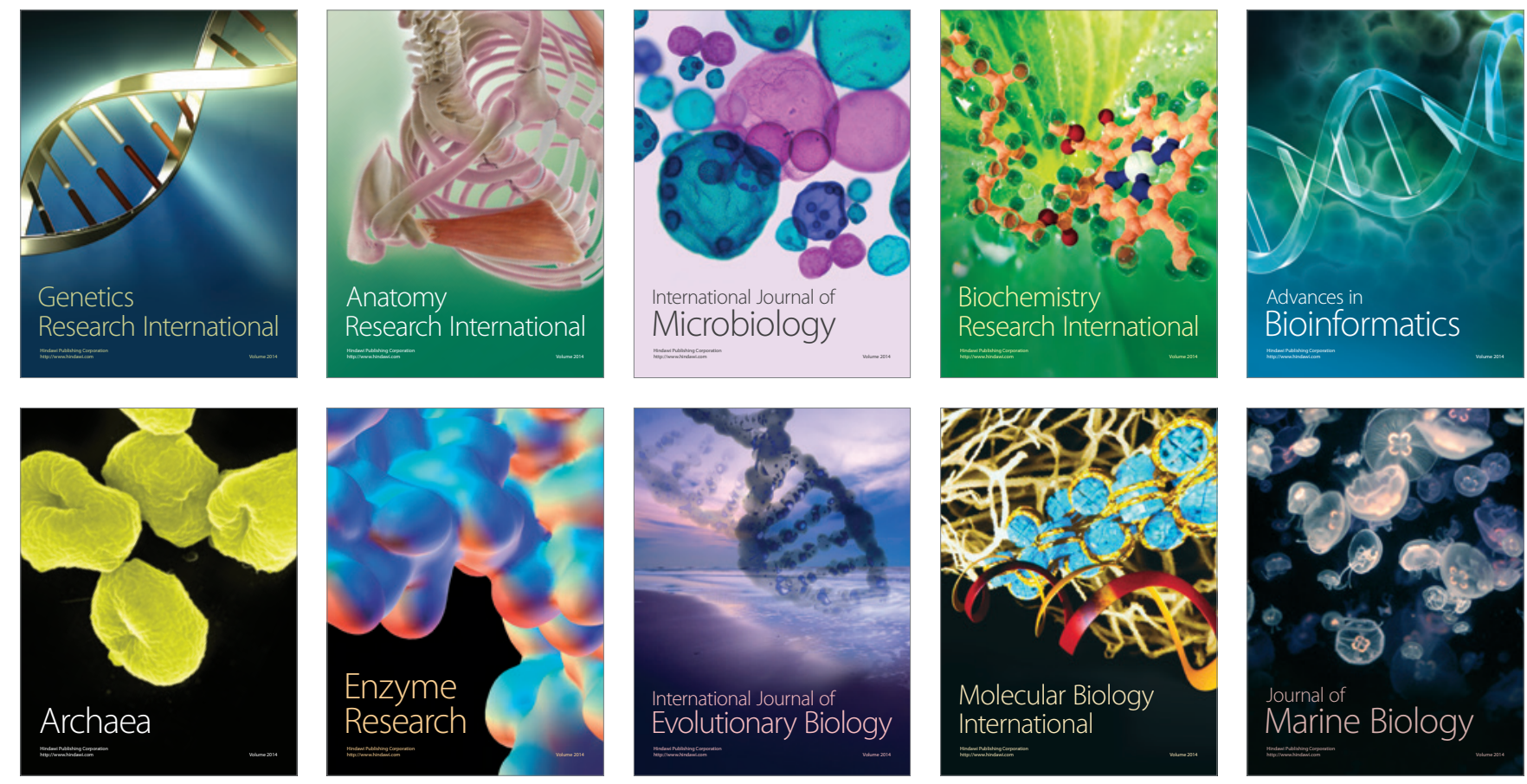